\title{
Efectos biológicos y uso herbolario del género Croton. Revisión sistemática
}

\author{
Biological effects and traditional use of genus Croton. A systematic review
}

Guadalupe C. Romero-Juárez ${ }^{a}$, Mario I. Ortiz ${ }^{b}$ y Raquel Cariño-Cortés ${ }^{c}$

\begin{abstract}
:
Background

Since ancient times, human beings have had the need to seek natural alternatives to combat various diseases. The active ingredients of the genus Croton (family Euphobiaceae) are used as natural pharmaceutical alternatives in African, South Asia and South America traditional medicine. Specifically, Croton hypoleucus is used in Mexican traditional medicine for the treatment of stomachache, gastritis, cough and local infections. Therefore, the main objective of the present review is to show the biological activities and clinical uses of Croton.
\end{abstract}

\section{Method}

A PubMed, Scopus, Web of Science literature search was performing. We reviewed the experimental studies of in vitro and in vivo models of the components of different Croton species published from 1980 to 2019, where each article synthesizes the information on the efficacy and biological effects of each isolated compound.

\section{Results}

A total of 16 experimental studies were included. No experimental clinical study was found in the literature. Low to moderate cytotoxic activity against different cell lines was produced by some species of croutons. These effects were mostly attributed to diterpenoids, crotonides and mostly clerodanes. A pure extract of Croton hypoleucus demonstrated moderate hepatoprotective and antioxidant activity.

\section{Conclusion}

Literature indicates the possible mechanism of action of some biological activities of Croton compounds. However, the information available is scarce. Therefore, studies in different experimental models are needed to clarify the security and efficacy of its active components in order to have natural alternatives with the same or better effect that conventional medications.

\section{Keywords:}

Croton, clerodane, folk medicine, biological effects, tenet active

\section{Resumen:}

\section{Antecedentes}

Desde la antigüedad, los seres humanos han tenido la necesidad de buscar alternativas naturales para combatir diversas enfermedades. Los principios activos de géneros de Croton (familia Euphobiaceae) se usan como alternativas terapéuticas naturales en la medicina tradicional en África, sur de Asia y Sudamérica. Específicamente, Croton hypoleucus (Ch) se usa en la medicina tradicional mexicana

\footnotetext{
a Universidad Autónoma del Estado de Hidalgo, Área Académica de Biología, Centro de Investigación Biológica, https://orcid.org/00000001-6903-3183, Email: ro362386@uaeh.edu.mx

b Autor de Correspondencia, Universidad Autónoma del Estado de Hidalgo, Área Académica de Medicina, Instituto de Ciencias de la Salud, https://orcid.org/0000-0003-1047-6304, Email: mario_i_ortiz@hotmail.com

c Universidad Autónoma del Estado de Hidalgo, Área Académica de Medicina, Instituto de Ciencias de la Salud, https://orcid.org/0000-
} 0003-4776-3534, Email: raquelcarcortes@gmail.com 
para el tratamiento del dolor de estómago, gastritis, tos e infecciones locales. Por tanto, el objetivo principal de la presente revisión es mostrar las actividades biológicas y los usos clínicos de Croton.

\section{Método}

Se realizó una búsqueda bibliográfica en PubMed, Scopus, Web of Science. Revisamos los estudios experimentales de modelos in vitro e in vivo de los componentes de las diferentes especies de Croton publicados de 1980 a 2019, donde de cada artículo se sintetizó la información sobre la eficacia y los efectos biológicos de cada compuesto aislado.

\section{Resultados}

Se incluyeron un total de 16 estudios experimentales. No se encontraron estudios clínicos en la literatura. Algunas especies de croton produjeron actividad citotóxica de baja a moderada contra diferentes líneas celulares. Estos efectos se atribuyen principalmente a diterpenoides, crotónidos y principalmente a clerodanos. Un extracto puro de Croton hypoleucus demostró moderada actividad hepatoprotectora y antioxidante.

\section{Conclusión}

La literatura indica el posible mecanismo de acción de algunas actividades biológicas de los compuestos de Croton. Sin embargo, la información disponible es escasa. Por lo tanto, se necesita realizar más estudios en diferentes modelos experimentales para clarificar la seguridad y eficacia de sus componentes activos con el fin de tener alternativas naturales con el mismo efecto o mejor que los medicamentos convencionales.

\section{Palabras Clave:}

Croton, clerodano, medicina tradicional, efectos biológicos, principio activo

\section{Introducción}

La diversidad de flora mexicana juega un papel importante en la producción agrícola, alimenticia, producción de materias primas para la industria e inclusive como una alternativa terapéutica eficaz. 1 Esta última, en la actualidad ha tomado gran importancia dentro de la salud humana. En este sentido, alrededor de 4,000 especies de plantas con flores (aproximadamente $15 \%$ de la flora total), partes de ellas o metabolitos secundarios con principios activos específicos, se les ha atribuido un efecto curativo de distintas enfermedades como diabetes, hipertensión, cáncer, dolor, infecciones locales, entre otras.2,3

Específicamente, el género Croton, el cual pertenece a la familia Euphobiaceae es empleada en la medicina tradicional en África, sur de Asia y Sudamérica para tratar diversos padecimientos. Este género de aproximadamente 1,300 especies 4-6,7, están distribuidas ampliamente en regiones tropicales y subtropicales de México, y con una mayor distribución en el suroeste de China, Vietnam, Laos y Tailandia.5-7 En general, las partes aéreas y/o raíz de las diferentes especies de Croton son usadas para tratar padecimientos relacionados con mordeduras de serpientes, dolor de estómago, abscesos, malaria, inflamación, hipertensión, diabetes e incluso cáncer.8-26 Para el caso del territorio mexicano, se localizan alrededor del $10 \%$ de las especies de Croton existentes en el mundo.7 Esta revisión tiene como objetivo mostrar las actividades biológicas y usos clínicos del género Croton.

\section{Métodos}

El objetivo de esta revisión es mostrar las actividades biológicas y usos clínicos del género Croton.

Se llevó a cabo una búsqueda bibliográfica en PubMed, Scopus, Web of Science, de 1989 a 2019, cuidando que el $80 \%$ de la bibliografía seleccionada fuera en idioma inglés. La búsqueda se llevó acabo utilizando palabras claves tales como [ "Euphobiaceae"], ["Clerodanos" ], [ "Crotonidos" ], [ "Croton "O" Especies de Croton" ], [ "Clerodanos" ], [ "Efectos biológicos de Croton" ] y [ "Plantas medicinales" ]. La revisión bibliográfica buscó identificar estudios experimentales realizados de distintas especies de Croton y sus efectos biológicos in vitro e in vivo. Asimismo, se buscó evidencia experimental de su uso en padecimientos o enfermedades en humanos.

\section{Resultados}

Se registró un total de 16 artículos, los cuales se revisaron de forma exhaustiva identificando los principales compuestos y/o metabolitos secundarios, así como, sus efectos biológicos o farmacológicos. 
De la literatura citada, ninguna de ellas siguió un modelo experimental clínico. Sin embargo, esta revisión incluyó estudios experimentales en modelos in vitro realizados sobre once especies de Croton distribuidas en el continente asiático (C. crassifolius, C. laevigatus, C. laui, C. glabellus, C. oblongifolius, C. sublyratus, C. insularis, C. membranaceus y $C$. euryphyllus)10-23 y adicionalmente, C. urucurana 24,25 y C. hypoleucus 26. Las especies descritas en la Tabla 1 revelan en general actividad citotóxica de baja a moderada frente a diferentes líneas celulares, donde Croton crassifolious, mostró actividad antiviral (herpes tipo l) $10 \mathrm{y}$ antiangiogénica 11, acción citotóxica moderada sobre células de carcinoma hepatocellular (HepG2 y Hep3B) y de colon de ratón (CT26 WT), así como contra leucemia promielocítica humana (HL-60) y de pulmón (A549) 12, 13; estas dos últimas líneas celulares también estudiadas con Croton laevigatus.14 Croton laui también mostró actividad citotóxica moderada en células HL- 60 y leucemia murina (P-388).15, 16. Además, esta última especie también presentó actividad antimicrobiana contra bacterias Gram positivas (Staphylococus aureus, Staphylococus epidermis, Micrococus lateus y Bacillus subtilis)17 y efecto antiinflamatorio en una línea celular murina (RAW 264.7).

Croton glabellus, presentó actividad antitumoral moderada en una línea celular de leucemia (K562), cáncer de colon (HCT15) y de sistema nervioso central (U251).18 Croton oblongifolius mostró actividad citotóxica contra cinco líneas celulares: carcinoma hepatocellular (HepG2), cáncer mamario humano (BT474), cáncer gástrico humano (KARO 3), carcinoma oncogénico (CHAGO) y cáncer de colon (SSW-620); cuyo efecto citotóxica fue mayor en cáncer mamario y de colon $\left(\mathrm{IC}_{50}\right.$ : 3.26-6.18 $\mu \mathrm{g} / \mathrm{mL} \quad$ y $\quad \mathrm{IC}_{50}: 4.16-5.20 \mu \mathrm{g} / \mathrm{mL}$, respectivamente). 19

Por su parte, Croton sublyratus, tuvo un efecto gastroprotector significativo (36-88\%).20 Igualmente, Croton insularis mostró un efecto citotóxico moderado en células de carcinoma cervical humano (HeLa) y leucemia de ratón (L1210) (91-200 $\mu \mathrm{M} \quad$ y 91-131 $\mu \mathrm{M}$, respectivamente).21 En cuanto a Croton membranaceus, el único compuesto citado (crotonmembranafurano) tuvo actividad citotóxica en células de cáncer de próstata (PC3). 18,22

Croton euryphyllus por su parte, mostró efecto citotóxico mínimo en una línea celular de tumor de médula suprarrenal (PC12) (7.14-9.72\%).23

Así mismo, Croton urucurana, tuvo efecto antiinflamatorio y antinociceptivo en un modelo de ratón (mus musculus albino) con eficacia de 41.9-63.8\%.24,25

Finalmente, con respecto a Croton hypoleucus, presentó actividad antioxidante y hepatoprotectora moderada in vivo.26
Los compuestos identificados en Croton sp., tales como clerodanos13,18,26, terpenos, diterpenoides, flavonoides, crotónidos 15-18,22-23, entre otros, han sido reportados debido a sus propiedades antibacterianas y antivirales 10 , citotóxicas 12,14-16,18-23 antiagiogénicas13, antiinflamatorias 17,24-25, antinociceptivas13,24-25 y hepatoprotectoras26; donde la mayor eficacia ante estos efectos biológicos se le atribuye a clerodanos y diterpenos. Por lo tanto, es necesario realizar más estudios que permitan confirmar los efectos encontrados in vitro, desde la perspectiva citotóxica, antinociceptiva y antiinflamatoria en modelos preclínicos.

\section{Discusión}

De las 16 referencias analizadas en esta revisión, nueve de las especies del género Croton corresponden a estudios in vitro10-23 y dos de ellas a estudios in vivo.2426

Dentro de los estudios in vitro, destacan los realizados en líneas celulares tumorales; se reportaron 17 líneas celulares, donde la eficacia en relación a las $\mathrm{IC}_{50}$ fue moderada. Las líneas celulares más estudiadas fueron HepG2 11,19, A549 y HL-60 13-15; de las cuales, $C$. oblongifolius presentó un mayor efecto citotóxico contra HepG2 ( $\mathrm{IC}_{50}$ : $\left.6.58 \mu \mathrm{g} / \mathrm{mL}\right)$ en comparación con $C$. crassifolius ( $\mathrm{IC}_{50}: 42.04 \mu \mathrm{M}-51.65 \mu \mathrm{M}$, respectivamente); igualmente, esta especie también mostró mejor actividad citotóxica contra A549 y HL-60 con $\mathrm{IC}_{50}: 8.4-11.8 \pm 2.1 \mu \mathrm{M}$ y $5.2 \pm 0.4-10.5 \mu \mathrm{M}$, respectivamente.

Respecto al único estudio antiviral, el tipo de virus frente al cual presentó eficacia $C$. crassifolius fue en Herpes tipo I (IC $50: 50,50,50,25$ y $50 \mu \mathrm{g} / \mathrm{mL}$ respectivamente). 10

Finalmente, los estudios in vivo, indican que la actividad antiinflamatoria que presentó $C$. laui 17 , tuvo menor efecto que $C$. urucurana con una eficacia de $41.9 \% 25$; por otra parte, el extracto puro de $C$. hypoleucus podría ser aparentemente inocuo en rata, ya que la dosis letal media $\left(L D_{50}\right)$ fue de $0.6307 \mathrm{mg} / \mathrm{mL}$. Además, se reportó que el extracto es eficaz frente un modelo experimental de daño hepático inducido por tioacetamida en ratas wistar.26 De acuerdo al único estudio fitoquímico de la especie antes mencionada, reportado por Velázquez-Jiménez y cols. 2018, crotonpenos (A y B) y tres nuevos epoxy clerodanos (hypolieins $A-C$ ) fueron aislados de las partes aéreas de $C$. hypoleucus.7 Los autores demostraron una actividad hepatoprotectora in vivo significativa, reduciendo los marcadores de dicho daño (28.13 $78.36 \%$ ), además de controlar moderadamente los niveles de defensa antioxidante in vitro (IC50 de 0.63 $\mathrm{mg} / \mathrm{ml}) .7$ Respecto a la toxicidad del extracto de $C$. 
hypoleucus utilizado, se reporta la supervivencia de todos los animales en todas las dosis evaluadas durante las semanas de estudio.7

La actividad hepatoprotectora y antioxidante que presenta el extracto de $C$. hypoleucus 26 , puede dilucidar que esta última podría estar relacionada con actividad antiinflamatoria. Sin embargo, los mecanismos de acción involucrados de los compuestos aislados de las diferentes especies de Croton incluidas en esta revisión aún no se conocen con claridad.

Tabla 1. Compuestos identificados / dosis administrada de las distintas especies de Croton, así como el modelo experimental aplicado para cada caso.

\begin{tabular}{|c|c|c|c|c|}
\hline Especie & $\begin{array}{c}\text { Modelo } \\
\text { experimental }\end{array}$ & $\begin{array}{l}\text { Compuestos identificados/ } \\
\text { Dosis administradas }\end{array}$ & Resultados & Ref \\
\hline $\begin{array}{l}\text { Croton } \\
\text { crassifolius }\end{array}$ & $\begin{array}{l}\text { a. Antiviral: } \\
\text { herpes tipo I } \\
\text { (HSV-1) y } \\
\text { b. adtotoxicid } \\
\text { ad y } \\
\text { anticancerígeno: } \\
\text { carcinoma } \\
\text { hepatocelular } \\
\text { (HepG2 } \\
\text { Hep3B). } \\
\text { c. Citotoxicid } \\
\text { ad contra células } \\
\text { CT26.WT } \\
\text { (carcinoma de } \\
\text { colon de ratón). } \\
\text { d. Citotoxicid } \\
\text { ad contra líneas } \\
\text { celulares A549 y } \\
\text { HL- 60 Antiangiog } \\
\text { e. énico para la } \\
\text { énico de } \\
\text { formación d dasos }\end{array}$ & 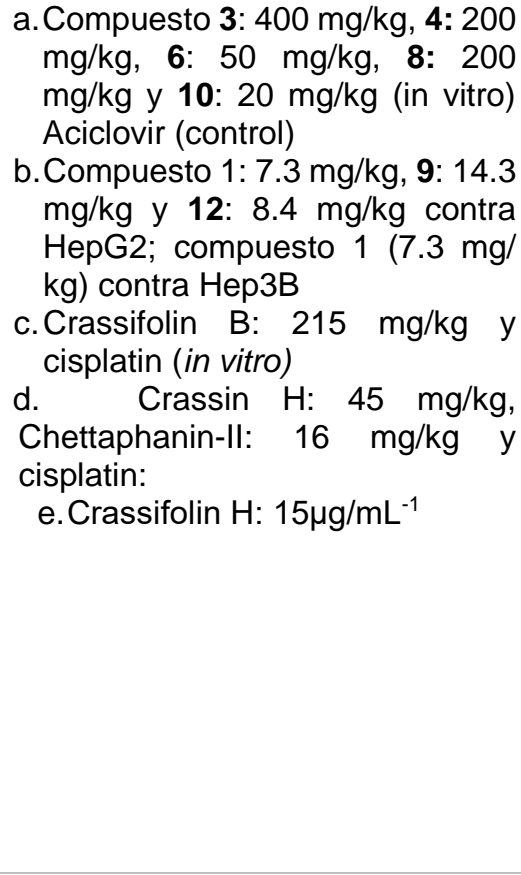 & 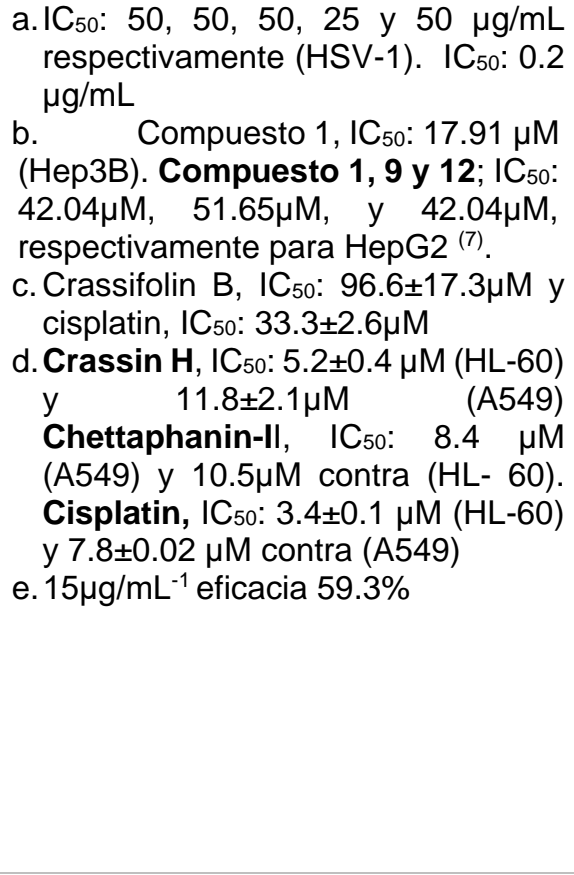 & $\begin{array}{l}10- \\
13\end{array}$ \\
\hline $\begin{array}{c}\text { Croton } \\
\text { laevigatus }\end{array}$ & $\begin{array}{lr}\text { a. } & \text { Antitumoral } \\
\text { contra } & \text { líneas } \\
\text { celulares } & \text { A549 y } \\
\text { HL-60 } & \end{array}$ & a. Levinoide A, $598 \mathrm{mg} / \mathrm{kg}$ & a. $598 \mathrm{mg} / \mathrm{kg}$, eficacia $0.012 \%$ & 14 \\
\hline Croton laui & $\begin{array}{l}\text { a. Citotoxicidad: } \\
\text { leucemia } \\
\text { promielocítica } \\
\text { humana (HL-60) y } \\
\text { P-388 (leucemia } \\
\text { murina). } \\
\text { b. Antimicrobial/ } \\
\text { antibacterial: } \\
\text { bacterias Gram- } \\
\text { positivas } \\
\text { C. Antiinflamatorio } \\
\text { in vitro: LPS- } \\
\text { stimulated RAW } \\
264.7 \text { cells }\end{array}$ & $\begin{array}{l}\text { a. Crotonólido A, } 3 \mathrm{mg} \\
\text { b. Crotonólido G, } 52 \mathrm{mg} \\
\text { c. Launina A: } 9.0 \mathrm{mg} \text {; Launina E: } \\
20 \mathrm{mg} \text {; Launina G: } 10 \mathrm{mg} \text {; Launina } \\
\text { H: } 5.5 \mathrm{mg} \text {; compuesto 14: } 11.5 \mathrm{mg}\end{array}$ & 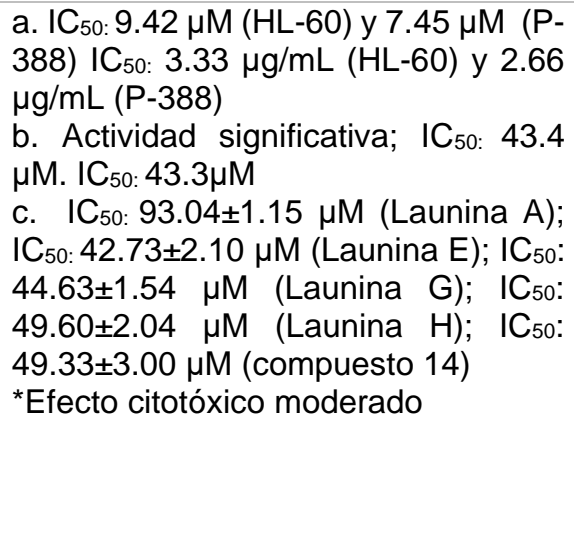 & $\begin{array}{l}15- \\
17\end{array}$ \\
\hline
\end{tabular}




\begin{tabular}{|c|c|c|c|c|}
\hline $\begin{array}{l}\text { Croton } \\
\text { glabellus }\end{array}$ & $\begin{array}{l}\text { a. Antitumoral: } \\
\text { leucemia (K562); } \\
\text { cáncer de colon } \\
\text { (HCT15); (U251) } \\
\text { sistema nervioso } \\
\text { central. }\end{array}$ & $\begin{array}{l}\text { a. Clerodane type diterpenes CG } \\
(1,6-8): 50 \mu \mathrm{mol} / \mathrm{L} \\
\text { b. } 270 \mathrm{~g} \text {, extracto crudo } \mathrm{CG} \\
\text { c. Compuesto } 2-4,9 \text { y adriamicin } \\
\text { (control) }\end{array}$ & 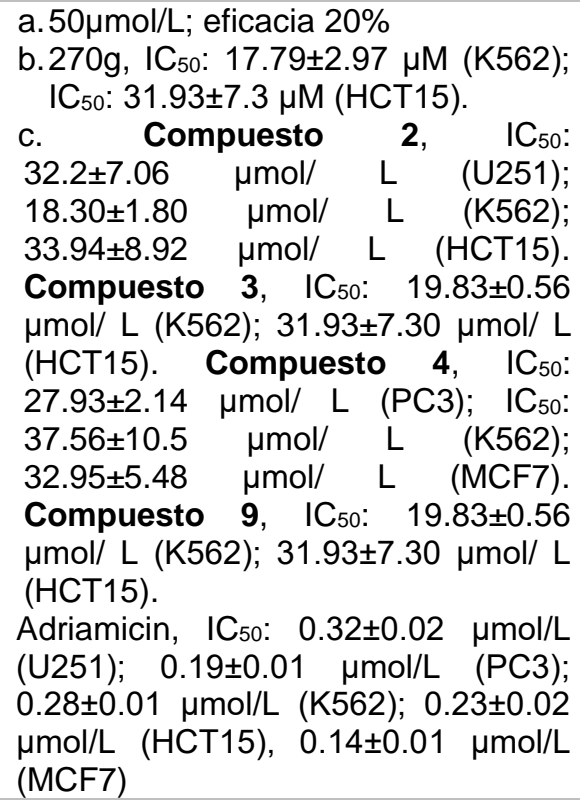 & 18 \\
\hline $\begin{array}{l}\text { Croton } \\
\text { oblongifoli } \\
\text { us }\end{array}$ & $\begin{array}{lr}\text { a. } & \text { Citotoxidad: } \\
\text { HepG2, } & \text { BT474, } \\
\text { KATO- r } & 3, \\
\text { CHAGO y } & \text { SW- } \\
620 & \end{array}$ & $\begin{array}{l}\text { a. Compuesto } 3: 22.4 \mathrm{mg} / \mathrm{kg} \text { (in } \\
\text { vitro) } \\
\text { b. Compuestos } 4-7: 25.0,8.5,53.2 \\
\text { y } 5.6 \mathrm{mg} / \mathrm{kg} \text { respectivamente }\end{array}$ & 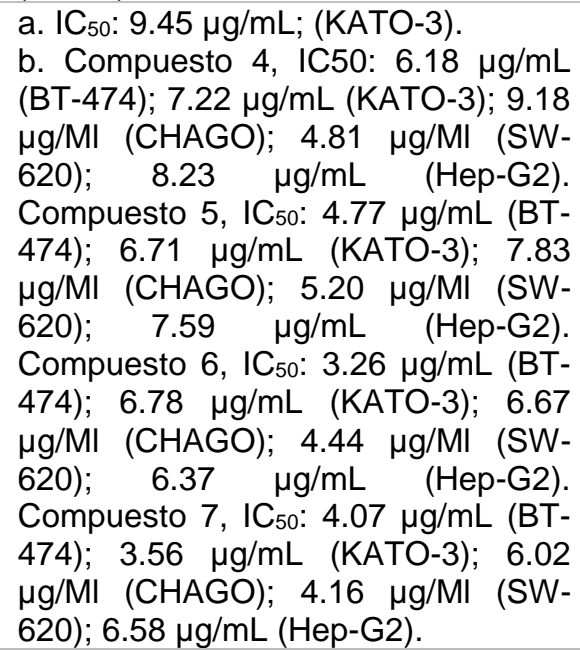 & 19 \\
\hline $\begin{array}{c}\text { Croton } \\
\text { sublyratus }\end{array}$ & $\begin{array}{l}\text { a. Citotoxici } \\
\text { dad gástrica (anti- } \\
\text { Shay ulcer } \\
\text { activity): Shay-rats }\end{array}$ & $\begin{array}{l}\text { a.Plaunol A:3/10mg/kg, i.p. } \\
\text { b. Plaunol B: } 3 / 10 \mathrm{mg} / \mathrm{kg} \text {, i.p } \\
\text { c. Plaunol C: } 3 / 10 \mathrm{mg} / \mathrm{kg} \text {, i.p } \\
\text { d. Plaunol D: } 3 / 10 \mathrm{mg} / \mathrm{kg} \text {, i.p } \\
\text { e. Plaunol E: } 3 / 10 \mathrm{mg} / \mathrm{kg} \text {, i.p }\end{array}$ & $\begin{array}{l}\text { a. } 3 / 10 \mathrm{mg} / \mathrm{kg} \text {, eficacia } 0 \% \\
\text { b. } 3 / 10 \mathrm{mg} / \mathrm{kg} \text {, eficacia } 55 \text { y } 85 \% \\
\text { c. } 3 / 10 \mathrm{mg} / \mathrm{kg} \text {, eficacia } 36 \text { y } 88 \% \\
\text { d. } 3 / 10 \mathrm{mg} / \mathrm{kg} \text {, eficacia } 44 \text { y } 61 \% \\
\text { e. } 3 / 10 \mathrm{mg} / \mathrm{kg} \text {, eficacia } 52 \text { y } 82 \% \\
{ }^{*} \text { Promedio de eficacia } 36-88 \%\end{array}$ & 20 \\
\hline $\begin{array}{l}\text { Croton } \\
\text { insularis }\end{array}$ & $\begin{array}{l}\text { Citotoxicidad: } \\
\text { carcinoma cervical } \\
\text { humano (HeLa) y } \\
\text { leucemia- mouse } \\
\text { (L1210) }\end{array}$ & $\begin{array}{l}\text { Compuesto } 1-6: 11.7,59.2,6.5 \text {, } \\
5.4,28.9 \text { mg respectivamente. }\end{array}$ & 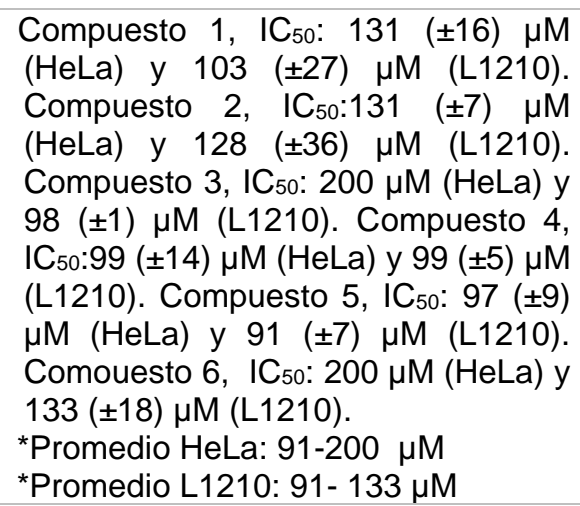 & 21 \\
\hline $\begin{array}{c}\text { Croton } \\
\text { membrana } \\
\text { ceus }\end{array}$ & $\begin{array}{l}\text { Citotoxicidad } \\
\text { contra PC-3 cells }\end{array}$ & Crotomembranafuran & $\mathrm{IC}_{50}: 10.6 \mu \mathrm{M}$ & 22 \\
\hline
\end{tabular}




\begin{tabular}{|c|c|c|c|c|}
\hline $\begin{array}{c}\text { Croton } \\
\text { euryphyllu } \\
s\end{array}$ & $\begin{array}{l}\text { Citotoxicidad: } \\
\text { PC12 cells }\end{array}$ & $\begin{array}{l}\text { Crotoeurins A-C; } 36,50 \text { y } 65 \mathrm{mg} \\
\text { respectivamente. }\end{array}$ & Eficacia del $9.72 \%, 14.90 \%$ y $7.14 \%$ & 23 \\
\hline $\begin{array}{l}\text { Croton } \\
\text { urucurana }\end{array}$ & $\begin{array}{l}\text { a. Antiinflam } \\
\text { atorio y } \\
\text { antinociceptivas; } \\
\text { swiss mice (Mus } \\
\text { musculus albino) }\end{array}$ & $\begin{array}{l}\text { a. } 25,100 \text { y } 400 \mathrm{mg} / \mathrm{kg} \text { de } \\
\text { CU (v.o.) }\end{array}$ & $\begin{array}{l}\text { a. } 25 \mathrm{mg} / \mathrm{kg} \text {, eficacia } 41.9 \% \\
\text { b. } \quad 100 \mathrm{mg} / \mathrm{kg} 42.9 \%(120 \mathrm{~min}) \\
\text { c. } \quad 400 \mathrm{mg} / \mathrm{kg} 63.8 \% \text { (30 min) }\end{array}$ & $\begin{array}{l}24, \\
25\end{array}$ \\
\hline $\begin{array}{c}\text { Croton } \\
\text { hypoleucus }\end{array}$ & $\begin{array}{l}\text { a. Daño } \\
\text { hepático (uso de } \\
\text { marcadores } \\
\text { moleculares ALT y } \\
\text { AST), ratas con } \\
\text { tioacetamina. } \\
\text { b. Actividad } \\
\text { antioxidante y } \\
\text { hepatoprtectora, } \\
\text { Wister- rats }\end{array}$ & $\begin{array}{l}\text { a. } 300 \mathrm{mg} / \mathrm{kg} \text { de } \mathrm{CH} \text { con } 6.6 \\
\text { mmol } / \mathrm{kg} \text { de TA (i.p.). } \\
\text { b. Actividad antioxidante, } \\
2 \text { a } 6 \mathrm{mg} / \mathrm{mL}(\mathrm{ECh}) \\
\text { c. Actividad } \\
\text { hetoprotectora; Primera fase: } \\
10,100 \text { y } 1000 \mathrm{mg} / \mathrm{kg} \text { de Ch } \\
\text { (extracto crudo). Segunda fase: } \\
1600,2900 \text { y } 5000 \mathrm{mg} / \mathrm{kg} \text { de Ch } \\
\text { (extracto crudo) (i.g.) }\end{array}$ & $\begin{array}{l}\text { a. ALT, eficacia } 40 \% \text {. AST, eficacia } \\
30 \% \\
\text { b. IC50: } 0.6307 \mathrm{mg} / \mathrm{mL} \text {, inhibición de } \\
28.13 \% \text { y } 78.36 \% \text { respectivamente }\end{array}$ & 26 \\
\hline
\end{tabular}

\section{Conclusión}

En la revisión sistemática se encontraron un total de 16 artículos del género Croton, donde la mayoría de ellos corresponde a información sobre estudios citotóxicos en líneas celulares en 9 de las 11 especies reportadas.

Por lo tanto, el género Croton representa una oportunidad y un reto para las futuras investigaciones tanto químicas, preclínicas y clínicas enfocadas a dilucidar efectos antiinflamatorios.

\section{Conflictos de interés}

Durante la elaboración de esta revisión, no hubo ningún conflicto de interés.

\section{Referencias}

[1] Molina Mendoza J, Galván Villanueva R, Patiño Siciliano A, Fernández Nava R. Plantas medicinales y listado florístico preliminar del municipio de Huasca de Ocampo, Hidalgo, México. Polibotánica. 2012; 34: 259- 291

[2] Gallegos Zurita M. Las plantas medicinales: principal alternativa para el cuidado de la salud, en la población rural de Babahoyo, Ecuador. An la Fac Med. 2016; 77(4): 327-32

[3] Ocegeda S, Moreno E, Koleff P. Plantas utilizadas en la medicina tradicional y su identificación científica. Biodiversitas. 2005; 62: 12 15

[4] Fuentes Cervantes I, Villavicencio Nieto M, Pérez Escandón B. Plantas medicinales de Omitlán, Hidalgo, México. Estudios científicos en el estado de Hidalgo y zonas aledañas. 2013; 17: 129
[5] Gordillo M. Los géneros de la familia Euphorbiaceae en México (parte A). An del Inst Biol Ser Botánica. 2002; 73: 155- 196

[6] Puebla P, Guerrero MF, Correa SX. Flavonoides del Género Croton. Cienc. Quim. Farm. 2004; 33: 59-69

[7] Velázquez-Jiménez R, Vargas-Mendoza D, Gayosso-de-Lucio JA, González-Montiel S, Villagómez-Ibarra JR. Three novel epoxyclerodanes bearing a furan ring from Croton hypoleucus. Phytochem Lett. 2018; 24: 21-26

[8] Xu WH, Liu WY, Liang Q. Chemical constituents from croton species and their biological activities. Molecules. 2018; 23(9): 2333

[9] Li R, Morris-Natschke SL, Lee KH. Clerodane diterpenes: Sources, structures, and biological activities. Natural Product Reports. 2016; 33: 1166- 1226

[10] Qiu M, Cao D, Gao Y, Li S, Zhu J, Yang B, et al. New clerodane diterpenoids from Croton crassifolius. Fitoterapia. 2016; 108: 81- 86.

[11] Yuan QQ, Tang S, Song W Bin, Wang WQ, Huang M, Xuan LJ. Crassins A-H, diterpenoids from the Roots of Croton crassifolius. J Nat Prod. 2017; 80: 254- 260

[12] Tian JL, Yao GD, Wang YX, Gao PY, Wang D, Li LZ, Song SJ. Cytotoxic clerodane diterpenoids from Croton crassifolius. Bioorganic and Medicinal Chemistry Letters. 2017; 27: 1237- 1242

[13] Wang GC, Li JG, Li GQ, Xu JJ, Wu X, Ye WC, et al. Clerodane diterpenoids from croton crassifolius. J Nat Prod. 2012; 75: 2188- 2192

[14] Wang GC, Zhang H, Liu HB, Yue JM. Laevinoids A and B: Two diterpenoids with an unprecedented backbone from Croton laevigatus. Org Lett. 2013; 15: 4880- 4883

[15] Yang L, Zhang YB, Chen LF, Chen NH, Wu ZN, Jiang SQ, et al. New labdane diterpenoids from Croton laui and their anti-inflammatory activities. Bioorganic Med Chem Lett. 2016; 26: 4687- 4691

[16] Liu CP, Xu JB, Zhao JX, Xu CH, Dong L, Ding J, et al. Diterpenoids from croton laui and their cytotoxic and antimicrobial activities. J Nat Prod. 2014; 77: 1013-1020 
[17] Bao H, Zhang Q, Ye Y, Lin L. Naturally occurring furanoditerpenoids: distribution, chemistry and their pharmacological activities. Phytochemistry Reviews. 2017; 16: 235- 270

[18] García A, Ramírez Apan T, Cogordan JA, Delgado G. Absolute configuration assignments by experimental and theoretical approaches of ent -labdane- and cis - ent -clerodane-type diterpenes isolated from Croton glabellus. Canadian Journal of Chemistry. 2007; 84: 1593- 1602

[19] Pudhom K, Sommit D. Clerodane diterpenoids and a trisubstituted furan from Croton oblongifolius. Phytochem Lett. 2011; 4: 147- 150

[20] Kitazawa E, Sato A, Takahashi S, Kuwano H, Ogiso A. Novel Diterpenelactones with Anti-peptic Ulcer Activity from Croton sublyratus. Chem Pharm Bull. 1980; 28: 227- 234

[21] Graikou K, Aligiannis N, Skaltsounis AL, Chinou I, Michel S, Tillequin F, et al. New Diterpenes from Croton insularis. J Nat Prod. 2004; 67: 685-688

[22] Addae Mensah I, Waibel R, Achenbach H, Muriuki G, Pearce C, Sanders JKM. A clerodane diterpene and other constituents of Croton membranaceus. Phytochemistry. 1989; 28: 2759- 2761

[23] Pan Z, Ning D, Wu X, Huang S, Li D, Lv S. New clerodane diterpenoids from the twigs and leaves of Croton euryphyllus. Bioorganic Med Chem Lett. 2015; 25: 1229- 1332

[24] Azab A, Nassar A, Azab AN. Anti-inflammatory activity of natural products. Molecules. 2016; 21: 1321

[25] Cordeiro KW, Felipe JL, Malange KF, Do Prado PR, De Oliveira Figueiredo P, Garcez FR, et al. Anti-inflammatory and antinociceptive activities of Croton urucurana Baillon bark. J Ethnopharmacol. 2016; 183: $128-135$

[26] Urrutia Hernández TA, Santos López JA, Benedí J, Sánchez Muniz FJ, Velázquez González C, De la O Arciniega M, Jaramillo Morales OA Bautista M. Antioxidant and Hepatoprotective Effects of Croton hypoleucus Extract in an Induced-Necrosis Model in Rats. Molecules. 2019; 24(14): 2533. 